\title{
Evolutionary aspects of the ZZ/ZW sex chromosome system in the Characidae fish, genus Triportheus. A monophyletic state and NOR location on the W chromosome
}

\author{
RF Artoni ${ }^{1}$ and LAC Bertollo ${ }^{2}$ \\ ${ }^{1}$ Universidade Estadual de Ponta Grossa, Campus de Uvaranas, Departamento de Biologia Geral, 84030-900 Ponta Grossa, PR, \\ Brazil; ${ }^{2}$ Universidade Federal de São Carlos, Departamento de Genética e Evolução, Caixa Postal 676, 13565-905 São Carlos, SP, \\ Brazil
}

Four species/populations of Triportheus, T. guentheri, T. cf. elongatus and $T$. paranense from different Brazilian hydrographic basins, were studied cytogenetically. All the species showed a similar karyotypic macrostructure, with a diploid chromosome number $2 n=52$ and a ZZ/ZW sex chromosome system. Besides silver- and fluorochrome-staining, the chromosome mapping of 18S rDNA was also investigated using a biotinylated probe. In spite of some variation in the number of the NORs, a major chromosome site was always present on the short arm of an autosomal pair. In addition, a characteristic rDNA site was also observed on the telomeric region of the $\mathrm{W}$ chromosome in the four species/populations. In Triportheus differential reduction in size and heterochromatin accumulation appear to be the main processes associated with the evolution of the sex $W$ chromosome. The location of rRNA genes on this chromosome may correspond to a plesiomorphic condition in the genus and, if so, predates to the sex chromosome system differentiation, with a possible influence in the initial steps of this process.

Heredity (2002) 89, 15-19. doi:10.1038/sj.hdy.6800081

Keywords: sex chromosomes; Ag-NORs; $18 \mathrm{~S}$ rDNA; Triportheus fish

\section{Introduction}

Triportheus is a genus of neotropical Characidae fish showing particular features in respect to sex chromosomes. Falcão (1988), described a ZZ/ZW sex chromosome system in four Brazilian species, three of them ( $T$. elongatus, T. flavus and T. albus) belonging to the Amazon basin and the other (T. signatus) belonging to the Paraná basin. In all the species, the $\mathrm{W}$ chromosome was characterized by a large amount of heterochromatin and a reduced size in relation to the $Z$ chromosome.

After this initial study, additional analyses have also shown such a sex chromosome system in other Triportheus species. This is the case of T. guentheri from the São Francisco river, Brazil, which had variation in the size of the W chromosome due to its amount of heterochromatin (Bertollo and Cavallaro, 1992) and T. paranense from the Paraná basin, Argentina (Sánchez and Jorge, 1999).

The distribution and differentiation of the ZZ/ZW system in Triportheus were recently reviewed by Artoni et al (2001), adding three new reports from different Brazilian hydrographic basins to the previous studies: T. cf. elongatus from the Araguaia river, $T$. paranense from Cuiabá river, and T. paranense from Paraguai river. Thus, all the species already analyzed (about $50 \%$ of the known Tripor-

Correspondence: RF Artoni, Universidade Estadual de Ponta Grossa, Campus de Uvaranas, Departamento de Biologia Geral, 84030-900 Ponta Grossa, PR, Brazil.E-mail: rfartoni@uol.com.br

Received 27 September 2001; accepted 22 January 2002 theus species) share the same sex chromosome system, which seems to be a fixed character in the genus. In addition, this $\mathrm{ZZ/ZW}$ sex system contrasts with those described for other groups of fish, such as Leporinus, Semaprochilodus and Parodon, in which the W chromosome is enlarged in size due to increased heterochromatin (Galetti Jr and Foresti, 1986; Feldberg et al, 1987; Moreira-Filho et al, 1993).

In this work the occurrence of rDNA sites on the $\mathrm{W}$ sex chromosome of Triportheus was verified, using fluorescence 'in situ' hybridization with a $18 \mathrm{~S}$ rDNA probe. A possible implication of this rDNA in the evolutionary process of the $\mathrm{ZZ/ZW}$ sex chromosome system is also discussed.

\section{Materials and methods}

\section{Samples and chromosome preparation}

Seventy-four wild individuals of Triportheus fish from different regions of Brazil were analyzed: 20 male and four female T. guentheri from the São Francisco river (Três Marias, Minas Gerais State), nine male and two female T. cf. elongatus from the Araguaia river (Barra do Garças, Mato Grosso State), 10 male and 24 female T. paranense from the Cuiabá river (Cuiabá, Mato Grosso State) and three male and two female T. paranense from the Paraguai river (Corumbá, Mato Grosso do Sul State).

Chromosome spreads were obtained by direct preparations from kidney cells (Bertollo et al, 1978) and by 
short-term culture of cells (Fenocchio et al, 1991), using stimulation of mitotic activity (Lee and Elder, 1980).

\section{C-banding and Ag-staining}

Constitutive heterochromatin (C-banding) was detected according to Sumner (1972), with the following modifications: 2-day-old slides were immersed in $0.2 \mathrm{~N} \mathrm{HCl}$ for $15 \mathrm{~min}$, and in $5 \%$ barium hydroxide at $42^{\circ} \mathrm{C}$ for $1 \mathrm{~min}$ $30 \mathrm{sec}$. The slides were then quickly washed in $0.2 \mathrm{HCl}$ and incubated in $2 \times \mathrm{SSC}$ at $60^{\circ} \mathrm{C}$ for $60 \mathrm{~min}$, followed by Giemsa staining (5\%) for $5 \mathrm{~min}$. After these five steps, slides were washed in deionized water and air-dried. Some preparations were also stained with the fluorochrome DAPI after the conventional C-banding method (C-DAPI), according to Souza and Moreira-Filho (1995).

Nucleolus organizing regions were detected by silver nitrate staining (Ag-NORs), according to Howell and Black (1980). Briefly, the slides were treated with a gelatin-silver nitrate solution, covered with a coverslip, incubated at $60^{\circ} \mathrm{C}$ for $3-5 \mathrm{~min}$, washed in deionized water and air-dried.

The best metaphases were photographed using a 25 ISO Kodak imagilink film.

\section{Quinacrine and chromomycin $\left(\mathrm{CMA}_{3}\right)$ staining}

Chromosome staining with the AT-rich quinacrine fluorochrome followed the method described by Schmid (1980). The slides were first submitted to a gradual ethanol series $(100 \%, 70 \%$ and $30 \%$ : $30 \mathrm{sec}$ each) and immersed in a quinacrine solution $(5 \mathrm{mg} / 100 \mathrm{ml})$ for $20 \mathrm{~min}$ at room temperature. Fluorescent signals for GCrich regions were obtained using chromomycin $A_{3}$, according to Schweizer (1976). The slides were treated with $0.5 \mathrm{mg} / \mathrm{ml} \mathrm{CMA}_{3}$ for $1 \mathrm{~h}$ and counterstained with $0.1 \mathrm{mg} / \mathrm{ml}$ distamycin A (DA) for $15 \mathrm{~min}$. The best metaphases were photographed using a 100 ISO Kodak TMAX film, with an appropriated excitation filter.

\section{Fluorescent in situ hybridization (FISH)}

A fluorescent 185 rDNA probe was used for the localization of the ribosomal cistrons on the chromosomes. This was obtained from the nuclear DNA of Salminus brasiliensis (Pisces, Characidae), using the primers NS1 5'GTAGTCATATGCTTGTCTC-3' and NS8 5'-TCCGCAG GTTCACCTACGGA-3' (Hizume, 1994). After agarose gel analysis, the PCR products were purified with the Sephaglas Band Prep Kit (Pharmacia Biotech). Probes were labelled with biotinylated uridine (BdUTP), using the 'nick translation' kit, according to manufacturer instructions (Boehringer). Hybridization was detected with avidin-FITC and the chromosomes conterstained with propidium iodide. Chromosomal figures were obtained with a Kodak Gold Plus film, ISO 400, using a standard blue excitation filter.

\section{Chromosomal analyses}

About 30 metaphases were analyzed for each specimen and those of better quality were employed for the karyotype organization. At least six karyotypes were analyzed for each sex from each species/population. The arm ratio (Levan et al, 1964) was used to classify the chromosomes according their morphology, through standardized measurements.

\section{Results}

\section{Standard karyotype of Triportheus}

All the Triportheus species/populations analyzed presented the diploid chromosome number $2 n=52$ for both sexes, and female heterogamety related to a $\mathrm{ZZ/ZW}$ sex chromosome system (Figures 1 and $2 a, b$ ). The $Z$ chromosome corresponds to the biggest metacentric in the karyotype and the $W$ to a medium-sized submetacentric chromosome.

\section{C-banding}

Almost all the chromosomes of the complement showed heterochromatin on the pericentromeric region. In the $\mathrm{Z}$ chromosome a clear C-band on both telomeric regions was also detected, while the $\mathrm{W}$ chromosome appeared strongly heterocromatic (Figure 1b).

\section{NOR sites}

The nucleolar organizer region (Ag-NOR), which is located on the short arm of a characteristic autosomal pair, showed a size polymorphism in all the species (Figures 1c and 2c (g-1)). This same chromosome presented C-positive bands on each side of the NOR site (Figures $1 b$ and $2 c(h)$ ).

Sometimes additional Ag-NORs were found on the short arm of another unidentified autosome and on the long arm of the W chromosome. This occurs, for example, in T. paranense from the Cuiabá river (Figure 1c). In addition, a sporadic silver-stained region was also seen on the $\mathrm{Z}$ chromosome of T. guentheri (Figure 2c (c)).

Analysis of the NORs by FISH, using the 18S rDNA probe, confirmed the autosomal rDNA sites, as well as their location on the $W$ chromosome (Figure $2 b$ ), in all the species/populations analyzed. These rDNA sites were the only chromosome regions showing positive and negative signals after chromomycin $\mathrm{A}_{3}$ (Figure 2d) and quinacrine (Figure 2a) staining, respectively.

\section{Discussion}

Triportheus represents a genus of fish showing a constant diploid number $(2 n=52)$ and a relatively conserved karyotypic macrostructure, as well as a differentiated $\mathrm{ZZ/ZW}$ sex chromosome system (Artoni et al, 2001). While the $Z$ is a conservative chromosome, corresponding to the biggest metacentric in the karyotype (Figure 1a), the $W$ chromosome shows interspecific variations concerning its size, morphology and amounts of hetero- and euchromatin, being also largely heterochromatic (Figure 1b) and reduced in size in relation to the $\mathrm{Z}$ chromosome (Falcão, 1988; Artoni et al, 2001; present study). However, some variation in the degree of heterochromatinization of the W chromosome can be found among the Triportheus species (Artoni et al, 2001). The euchromatic regions of the $\mathrm{W}$, although very reduced in some species, could represent chromosomal segments that still maintain a homology with the $\mathrm{Z}$ chromosome, as well as the physical locations of the genes for sex determination.

Base specific fluorochromes were not efficient in detecting different DNA classes in the Triportheus karyotype, with the exception of NORs. These regions appear as GC-rich according to the chromosome staining with chromomycin and quinacrine. Indeed, NORs have been 


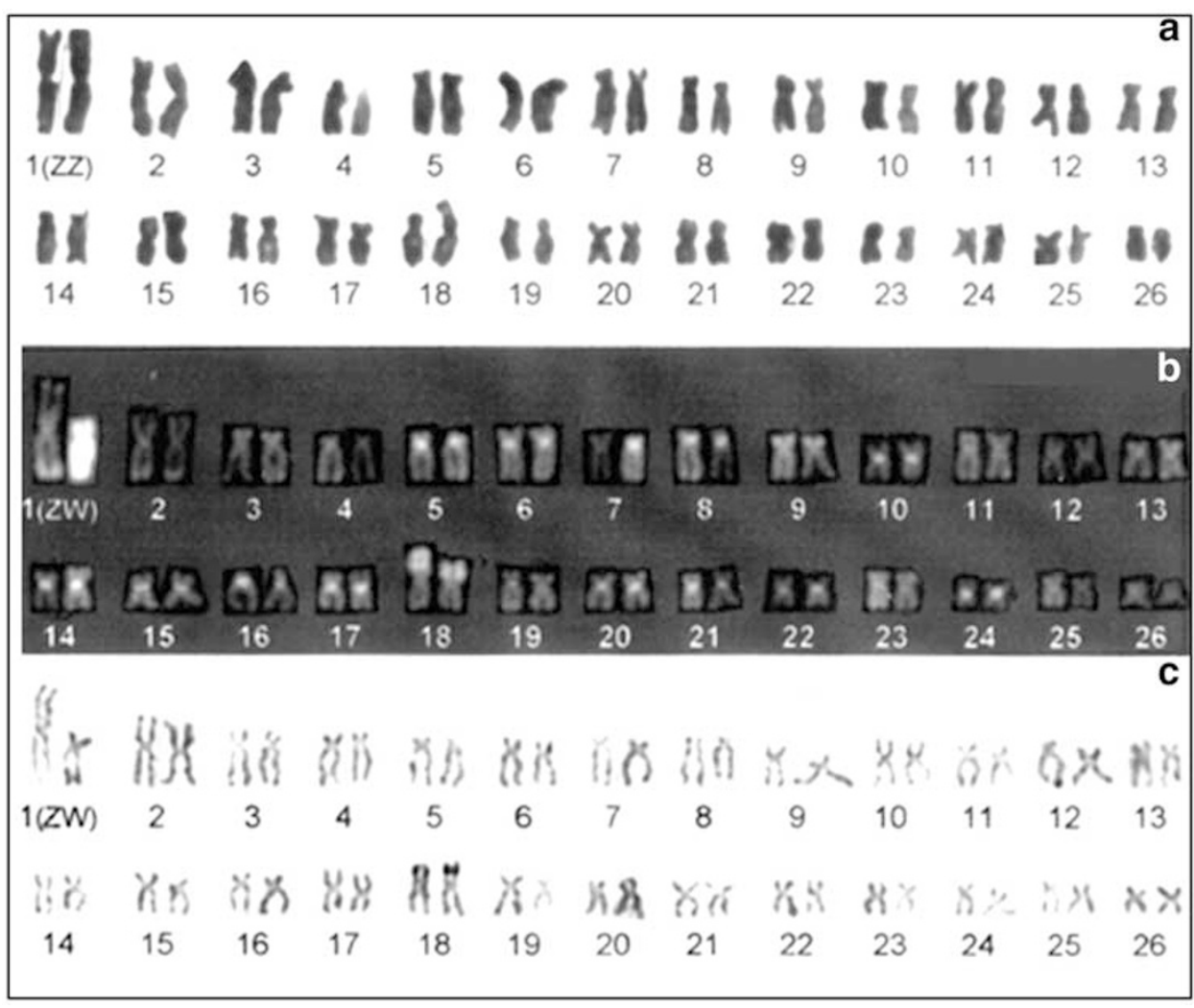

Figure 1 Male (a) and female (b, c) karyotypes of Triportheus paranense from the Cuiabá river, representatives for a general Triportheus karyotypic macrostructure. Conventional Giemsa staining (a), C-DAPI staining (b) and Ag-NORs (c).

characterized as GC-rich regions in lower vertebrates, such as amphibians (Schmid, 1980) and fishes (Amemyia and Gold, 1986; Phillips et al, 1988; Galetti Jr and Rasch, 1993a, b; among others). However, GC-rich heterochromatic segments not related to the NORs can also be seen in some fish species (Artoni et al, 1999).

A characteristic chromosome, bearing a secondary constriction on its short arm, appears to be a homeologous pair among the Triportheus species, showing a constant Ag-NOR (Figure 1). Sometimes another unidentified autosome pair was also stained with silver nitrate. Species with multiple NORs are not rare among fish (eg, Bertollo, 1996; Almeida-Toledo, 1998; Galetti Jr, 1998). In these cases numerical variation in Ag-NORs is frequently observed, which can be related to some factors, such as differential gene activation. The 'in situ' hybridization with a $18 \mathrm{~S}$ rDNA probe was an important tool for the NORs studies in Triportheus. Thus, a maximum of four hybridization signals were observed in the autosomes, two of them not always detected due their small size. Additionally, a typical positive signal was always seen in the female specimens, on the long arm of the $\mathrm{W}$ chromosome (Figure $2 \mathrm{~b}$ ). This region is labelled by chromomycin $\mathrm{A}_{3}$ (Figure 2d) and eventually is also stained with silver nitrate. No hybridization signal was detected in the $\mathrm{Z}$ chromosome, in spite of the sporadic occurrence of a silver-stained region on its long arm, as was seen in $T$. guentheri (Figure 2c (c)). This region may correspond to an argentophylic heterochromatin (Sumner, 1990), without any relationship to NORs, as observed in the Hoplias lacerdae fish group (Morelli, 1998).

Few examples of sex chromosomes bearing NORs are known in mammals, especially those on the $X$ chromosome, as in the bat Carollia perspecillata (Goodpasture and Bloom, 1975; Morielle and Varella-Garcia, 1988) and in the rodent Akodon arviculoides (Yonenaga-Yassuda et al, 1983). These species do not show a significant difference in the number of active rRNA genes among males and females. A similar situation occurs in the amphibian Gastrotheca riobambae, where the females (XX) show two NORs and the males (XY) just one, without any evidence for a dosage compensation (Schmid et al, 1983). Besides Tripotheus, NORs located on fish sex chromosomes were already found in Fundulus diaphanus (Howell and Black, 1979), in the Arctic char Salvelinus alpinus (Reed and Phillips, 1997), and Hoplias malabaricus (Born and Bertollo, 2000).

Among invertebrates, such as Drosophila and many Coleoptera, where the synaptonemal complex does not occur between the $\mathrm{X}$ and $\mathrm{Y}$ chromosomes, the synapsis is assured by the association between the rDNA present in these chromosomes, allowing a regular segregation during gametogenesis (Irick, 1994; Ren et al, 1997). Stitou et al (1997) also described latent NORs in the sex chromosomes of the rodent Lemniscomys barbarus, suggesting some role for the rDNA during the synapsis process of the $X$ and $Y$ chromosomes. In this species, this kind of DNA was observed only in one population and a fast chromosomal evolution through translocations events (autosome/sex chromosomes) was proposed. However, in Triportheus, a peculiar condition can be characterized in view of the occurrence of inactive/active NORs on the $W$ chromosome of the four species/populations analyzed. Thus, the location of rDNA sites on the sex chromosomes 

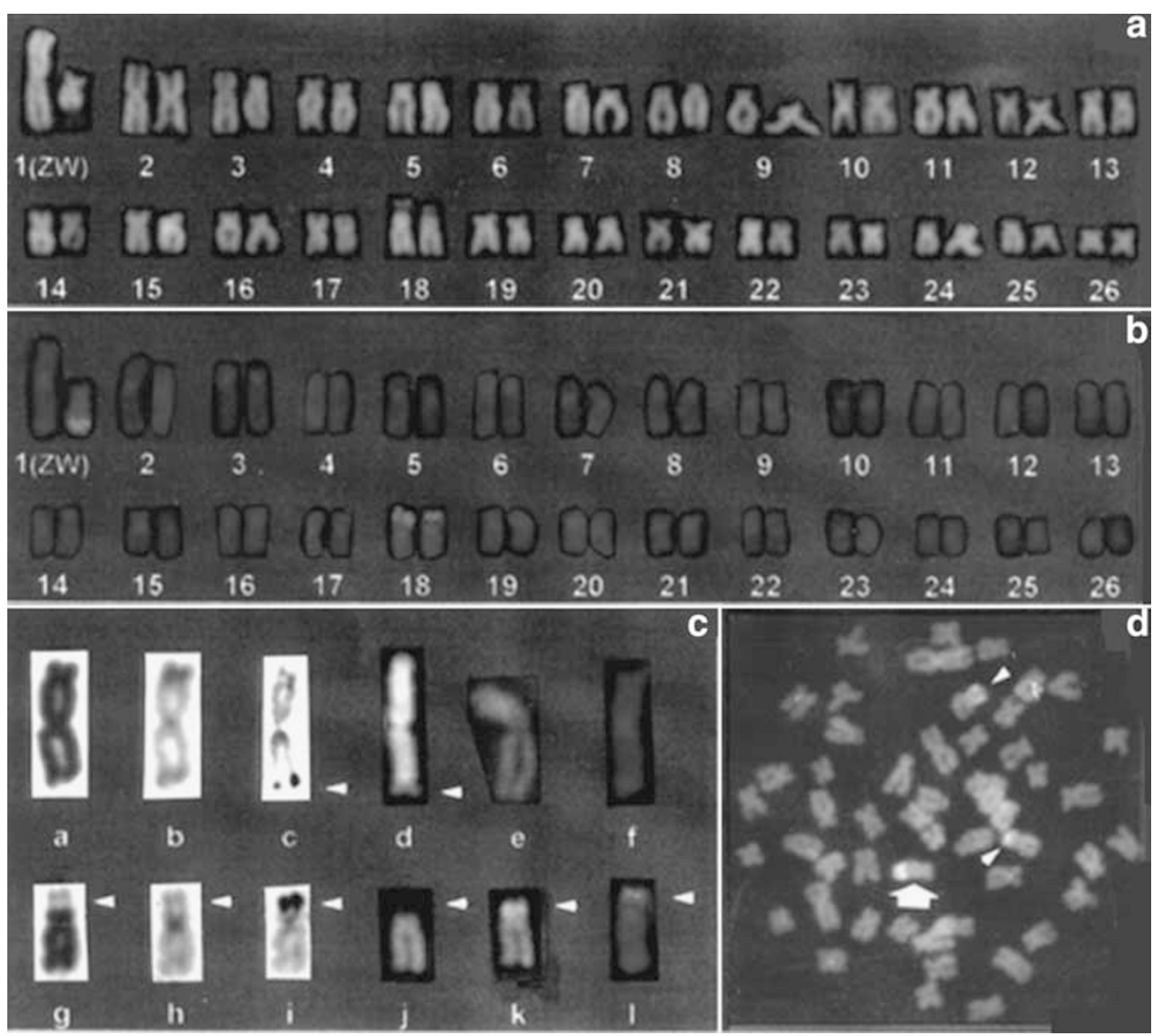

Figure 2 Karyotypes and chromosomes of Triportheus paranense from the Cuiabá river $(\mathbf{a}, \mathbf{b}, \mathbf{d})$ and T. guentheri from the São Francisco river (c). Quinacrine staining (a) and fluorescence 'in situ' hybridization with $18 \mathrm{~S} \mathrm{rDNA}$ probe (b) showing differential signals on the long arm of the W chromosome and on the short arm of the 18th autosomal pair. Chromomycin-stained metaphase (d) showing labelled NORs in the 18th pair (arrowheads) and in the $\mathrm{W}$ chromosome (arrow). In (c) the $\mathrm{Z}$ chromosome (a-f) and the major NOR bearing autosome (g-1) of T. guentheri are shown, after conventional Giemsa staining (a,g), C-banding (b,h), silver staining (c,i), quinacrine staining (d,j), chromomycin $\mathrm{A}_{3}$ staining $(\mathrm{e}, \mathrm{k})$ and FISH with $18 \mathrm{~S}$ rDNA probe $(\mathrm{f}, 1)$. The arrowheads indicate a sporadic silver-stained region on the $\mathrm{Z}$ chromosome and the NOR location on the autosome.

of Triportheus appears to be an old condition and probably already present in the ancestral $\mathrm{W}$ chromosome.

Some animal species offer special conditions for the study of sex chromosome differentiation. In the cassowary Casuarius casuarius (Aves, Ratitae), for instance, it was showed that the $W$ chromosome is at an early stages of differentiation from the $\mathrm{Z}$ chromosome and that structural rearrangements might have been the initial step of this differentiation from an ancestral homomorphic pair. FISH analyses showed that some loci are conservative on both $\mathrm{Z}$ and $\mathrm{W}$ chromosomes, while others are missing from the $\mathrm{W}$ chromosome, probably due to deletions in its proximal region, leading to differences in the size and banding pattern between the $\mathrm{Z}$ and $\mathrm{W}$ chromosomes. Thus, these results favour previous propositions that heterochromatinization was not the initial step in the differentiation of the ratite $\mathrm{W}$ chromosome (Nishida-Umehara et al, 1999).

However, in several neotropical fish species, heterochromatinization is thought to be a plausible hypothesis concerning the evolution of the ZW sex chromosome system (Galetti Jr and Foresti, 1986; Moreira-Filho et al, 1993; Artoni et al, 2001). The same is true for the Triportheus species, in which the heterochromatinization might have driven the differentiation of the $\mathrm{W}$ chromosome and its additional size reduction in respect to the $\mathrm{Z}$ chromosome (Artoni et al, 2001). In the Arctic char, Salvelinus alpinus, an important role was suggested for the rDNA loci on the putative sex chromosomes of this species, which might limit the opportunity for additional recombination near a major sex-determining locus, if this locus is adjacent to the rDNA sites (Reed and Phillips, 1997). In a similar way, we cannot discard a possible role for the repetitive DNA associated with the NORs, or even for the proper multicopy rDNA, in the differentiation of the sex chromosome system of Triportheus.

The ZZ/ZW sex chromosome system appears to be a synapomorphy in the Triportheus genus, representing a particular characteristic of this fish group among the large family Characidae. All of the nine species/populations already analyzed present a well differentiated ZZ/ZW system (Artoni et al, 2001). Accordingly, the occurrence of rDNA on the the W chromosome of this group may also correspond to a synapomorphic condition. Indeed, four different species/populations, from distinct hydrographic basins, present rDNA sites on an equivalent location in the $W$ chromosome. Thus, further studies with other Triportheus species will offer additional support to the present proposition, as well as to the chromosomal evolution of this fish group. 


\section{Acknowledgements}

The authors thank Dr MC Malabarba (Zoology Museum, PUC - Porto Alegre) for the identification of the species and Drs Yoshimi Sato (CODEVASF, Minas Gerais), Paulo C Venere (UFMT-ICLMA) and Carlos S Myiazawua (UFMT) for their help in supplying fishes. They also thank Issakar L Souza (MSc) for his help with the fluorescence 'in situ' hybridization. This work was supported by Conselho Nacional de Desenvolvimento Cientifico e Tecnológico (CNPq), Coordenação de Aperfeiçoamento de Pessoal de Nivel Superior (CAPES) and Fundação de Amparo a Pesquisa de Estado de São Paulo (FAPESP, proc. no. 96/02266-0).

\section{References}

Amemiya CT, Gold JR (1986). Chromomycin A stains nucleolus organizer regions of fish chromosomes. Copeia 1: 226-231.

Almeida-Toledo LF (1998). Cytogenetic markers in neotropical freshwater fishes. In: Malabarba LR, Reis RE, Vari RP, Lucena ZM, Lucena CAS (eds) Phylogeny and Classification of Neotropical Fishes, Edipucrs: Porto Alegre. pp 583-588.

Artoni RF, Molina WF, Bertollo LAC, Galettii JR PM (1999). Heterochromatin analysis in the fish species Liposarcus anisitsi (Siluriformes) and Leporinus elongatus (Characiformes). Genet Mol Biol 22: 1-6.

Artoni RF, Falcão JN, Moreira-Filho O, Bertollo LAC (2001). An uncommon condition for a sex chromosome system in Characidae fish. Distribution and differentiation of the $\mathrm{ZZ} / \mathrm{ZW}$ system in Triportheus. Chromosome Res 9: 449-456.

Bertollo LAC (1996). The nucleolar organizer regions of Erythrinidae fish. An uncommon situation in the genus Hoplias. Cytologia 61: 75-81.

Bertollo LAC, Takahashi CS, Moreira-Filho O (1978). Cytotaxonomic considerations on Hoplias lacerdae (Pisces, Erythrinidae) Brazil J Genet 1: 103-120.

Bertollo LAC, Cavallaro ZI (1992). A highly differentiated ZZ/ZW sex chromosome system in a Characidae fish Triportheus guentheri. Cytogenet Cell Genet 60: 60-63.

Born GG, Bertollo LAC (2000). An XX/XY sex chromosome system in a fish species, Hoplias malabaricus, with a polymorphic NOR-bearing $X$ chromosome. Chromosome Res 82: 111-118.

Falcão JN (1988). Caracterização cariotípica em peixes do gênero Triportheus (Teleostei, Characiformes, Characidae). PhD Thesis. Universidade de São Paulo, SP, Brazil.

Feldberg E, Bertollo LAC, Almeida-Toledo L, Foresti F, MoreiraFilho O (1987). Biological aspects of Amazonian fishes. IX. Cytogenetic studies in two species of the genus Semaprochilodus (Pisces, Prochilodontidae). Genome 29: 1-4.

Fenocchio AS, Venere PC, Cesar AG, Dias AL, Bertollo LAC (1991). Short term culture from solid tissues of fishes. Caryologia 44: 161-166.

Galetti Jr, PM (1998). Chromosome diversity in Neotropical fish. NOR studies. Ital J Zool 65 (Suppl): 53-56.

Galetti Jr, PM, Foresti F (1986). Evolution of the ZZ/ZW system in Leporinus (Pisces, Anostomidae). Cytogenet Cell Genet 43: 43-46.

Galetti Jr, PM, Rasch EM (1993a). NOR variability in diploid and triploid forms of the Amazon molly Poecilia formosa as shown by silver nitrate and chromomycin $\mathrm{A}_{3}$ staining. Brazil J Genet 16: 927-938.

Galetti Jr, PM, Rasch EM (1993b). Chromosome studies in Poecilia latipunctata with NOR polymorphism as shown by silver nitrate and chromomycin $\mathrm{A}_{3}$ (Teleostei, Poecilidae). Ichthyol Explor Freshwaters 4: 269-277.

Goodpasture C, Bloom SE (1975). Visualization of nucleolar organizer regions in mammalian chromosomes using silver staining. Chromosoma 53: 37-50.

Hizume M (1994). Allodiploid nature of Allium wakegi Araki revealed by genomic in situ hybridization and localization of $5 S$ and 18S rDNAs. Jap J Genet 69: 407-415.

Howell WM, Black DA (1979). Location of the nucleolus organizer regions on the sex chromosomes of the banded killifish Fundulus diaphanus. Copeia 4: 544-546.

Howell WM, Black DA (1980). Controlled silver staining of nucleolus organizer regions with a protective colloidal developer: a 1-step method. Experientia 36: 1014-1915.

Irick H (1994). A new function for heterochromatin. Chromosoma 103: 1-3.

Lee MR, Elder FFB (1980). Yeast stimulation of bone marrow mitosis for cytogenetic investigations. Cytogenet Cell Genet 26: 36-40.

Levan A, Fredga K, Sandberg AA (1964). Nomenclature for centromeric position on chromosomes. Hereditas 52: 201-220.

Moreira-Filho O, Bertollo LAC, Galetti Jr, PM (1993). Distribution of sex chromosome mechanisms in neotropical fish and description of a $\mathrm{ZZ/ZW}$ system in Parodon hilarii (Parodontidae). Caryologia 46: 115-125.

Morelli S (1998). Citogenética evolutiva em espécies do gênero Hoplias grupo lacerdae. Macroestrutura cariotípica, heterocromatina constitutiva e regiões organizadoras de nucléolo. PhD Thesis. Universidade Federal de São Carlos, SP, Brazil.

Morielle E, Varella-Garcia M (1988). Variability of nucleolus organizer regions in phyllostomid bats. Brazil J Genet 11: 853-871.

Nishida-Umehara C, Fujiwara A, Ogawa A, Mizuno S, Abe S, Yoshida MC (1999). Differentiation of $Z$ and W chromosomes revealed by replication banding and FISH mapping of sexchromosome-linked DNA markers in the cassowary (Aves, Ratitae). Chromosome Res 7: 635-640.

Phillips R, Pleyte KA, Hartley SE (1988). Stock-specific differences in the number and chromosome positions of the nucleolar organizer regions in Arctic char (Salvelinus alpinus). Cytogenet Cell Genet 48: 9-12.

Reed KM, Phillips RB (1997). Polymorphism of the nucleolus organizer region (NOR) on the putative sex chromosomes of Artic char (Salvelinus alpinus) is not sex related. Chromosome Res 5: 221-227.

Ren XJ, Eisenbauer L, Hong CS, Lee Y, McKee BD (1997). Roles of rDNA spacer and transcription unit-sequences in $X-Y$ meiotic chromosome pairing in Drosophila melanogaster males. Chromosoma 106: 29-36.

Sánchez S, Jorge LC (1999). A new report of the ZZ/ZW sex chromosome system in the genus Triportheus (Pisces, Triportheinae). Cytologia 64: 395-400.

Schmid M (1980). Chromosome banding in Amphibia IV. Differentiation of GC- and AT-rich chromosome regions in Anura. Chromosoma 77: 83-103.

Schmid M, Haaf T, Geile B, Sims S (1983). Chromosome banding in Amphibia. VIII. An unusual XY/XX sex chromosome system in Gastrotheca riobambae (Anura, Hylidae). Chromosoma 88: 69-82.

Schweizer D (1976). Reverse fluorescent chromosome banding with chromomycin and DAPI. Chromosoma 58: 307-324

Souza IL, Moreira-Filho O (1995). Cytogenetic diversity in the Astyanax scabripinnis species complex (Pisces, Characidae). I. Allopatric distribution in a small stream. Cytologia 60: 1-11.

Stitou S, Burgos F, Zurita F, Jiménez R, Sánches A, Diaz de la Guardia R (1997). Recent evolution of NOR-bearing and sex chromosome of the North African rodent Lemniscomys barbarus. Chromosome Res 5: 481-485.

Sumner AT (1972). A simple technique for demonstrating centromeric heterochromatin. Exp Cell Res 75: 304-306.

Sumner AT (1990). Chromosome Banding. Cambridge University Press: Cambridge.

Yonenaga-Yassuda Y, Assis FL, Kasahara S, L'Abbate M, Souza MJ (1983). Nucleolar organizer regions in Akodon arviculoides (Cricetidae, Rodentia): evidence for the activity of rDNA genes in both X chromosomes of females. Cytogenet Cell Genet 35: 143-147. 